\title{
Direct observation of domain-wall pinning at nanoscale constrictions
}

\author{
M. Kläui, ${ }^{a)}$ H. Ehrke, and U. Rüdiger \\ Fachbereich Physik, Universität Konstanz, Universitätsstraße 10, 78457 Konstanz, Germany \\ T. Kasama and R. E. Dunin-Borkowski \\ RIKEN, 2-1 Hirosawa, Wako, Saitama 381-0198, Japan and Department of Materials Science and \\ Metallurgy, University of Cambridge, Pembroke Street, Cambridge CB2 3QZ, United Kingdom \\ D. Backes ${ }^{\text {b) }}$ and L. J. Heyderman \\ Laboratory for Micro- and Nanotechnology, Paul Scherrer Institut, 5232 Villigen PSI, Switzerland \\ C. A. F. Vaz and J. A. C. Bland \\ Cavendish Laboratory, University of Cambridge, Madingley Road, Cambridge, CB3 OHE, \\ United Kingdom \\ G. Faini and E. Cambril \\ Laboratoire de Photonique et de Nanostructures-CNRS, Route de Nozay, 91460 Marcoussis, France \\ W. Wernsdorfer \\ Laboratoire L. Néel-CNRS, BP 138, 38274 Grenoble, France
}

(Received 23 June 2005; accepted 28 July 2005; published online 2 September 2005)

\begin{abstract}
In a combined experimental and numerical study, we determine the details of the pinning of domain walls at constrictions in permalloy nanostructures. Using high spatial-resolution $(<10 \mathrm{~nm})$ electron holography, we image the spin structure of geometrically confined head-to-head domain walls at constrictions. Low-temperature magnetoresistance measurements are used to systematically ascertain the domain-wall depinning fields in constrictions down to $35 \mathrm{~nm}$ width. The depinning fields increase from 60 to 335 Oe with decreasing constriction width and depend on the wall spin structure. The energy barrier to depin the wall from the constriction is quantitatively determined and comparison with the depinning field strength allows us to gauge the energy barrier height of the pinning potential.
\end{abstract}

The recent upsurge of interest in magnetic domain walls has been fuelled partly by a fundamental interest in the spin structure of nanoscale domain walls and in particular by possible novel logic and memory applications based on domain walls. ${ }^{1-3}$ Control of the domain wall behavior, and in particular of the magnetic switching, can be achieved through pinning centers, which provide well defined stable locations for doma in walls and can be used to confine the wall propagation. ${ }^{3-6}$ Pinning centers can result from imperfections in the material, ${ }^{7}$ which are inherently hard to control. Instead, artificially structured variations in the geometry of an element have been introduced to engineer such pinning. ${ }^{5,8-12}$ In addition to applications, such as domain-wall diodes in logic devices, ${ }^{8}$ constrictions have also allowed the determination of more fundamental properties of domain walls, such as magnetoresistance (MR) effects associated with domain walls and their quasiparticle spin-block behavior. ${ }^{10,11}$ To probe this landscape for the energy potential, rings have proven to be an apt geometry since, due to the curved geometry, domain walls can be positioned easily using uniform fields applied along appropriate directions. In earlier work on narrow rings, we observed indirectly by MR measurements that constrictions create an attractive potential well for transverse walls, but vortex walls are repelled from a constriction. ${ }^{10,11}$ While qualitatively the domain-wall behavior could be determined using MR measurements, the details of the wall positions and in particular the nanoscale wall spin

\footnotetext{
${ }^{\text {a)} E l e c t r o n i c ~ m a i l: ~ m a t h i a s @ k l a e u i . d e ~}$

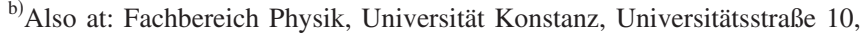
78457 Konstanz, Germany. structure-in structures with constrictions-has not been elucidated. An adequate characterization of this energy potential requires the determination of its width and amplitude, since for applications it is the strength of the pinning, which corresponds to the depth of the potential well, that is critical for engineering of devices with reliable switching, thermal stability, etc. From a fundamental point of view, the strength of the pinning is directly correlated with the wall spin structure, so that only the determination of its nanoscale spin structure will enable an in-depth understanding of the energetics governing the pinning strength.

In this letter, we image geometrically confined domain walls directly with sub-10 nm resolution using off-axis electron holography. We systematically determine the nanoscale spin structure of head-to-head and tail-to-tail domain walls in constrictions down to $35 \mathrm{~nm}$ width. We measure the magnetic fields that are needed to depin these domain walls from constrictions as a function of the constriction width and the domain-wall spin structure. From the nanoscale wall configuration, the energy barrier to depin the wall from a constriction is quantitatively determined and correlated with the experimentally measured depinning fields.

For magnetic induction mapping using electron holography, we have fabricated $200 \mathrm{~nm}$ wide permalloy $\left(\mathrm{Ni}_{80} \mathrm{Fe}_{20}\right)$ structures with constrictions by electron-beam lithography, molecular-beam epitaxy, and lift-off on $50 \mathrm{~nm}$ thick $\mathrm{SiN}$ membranes. ${ }^{13}$ A scanning electron microscopy (SEM) image of such a structure is presented in Fig. 1. Since for the fragile membranes the lift-off process cannot be assisted by ultrasonic agitation, wavy lines consisting of half-rings were used rather than complete ring structures for which the center 


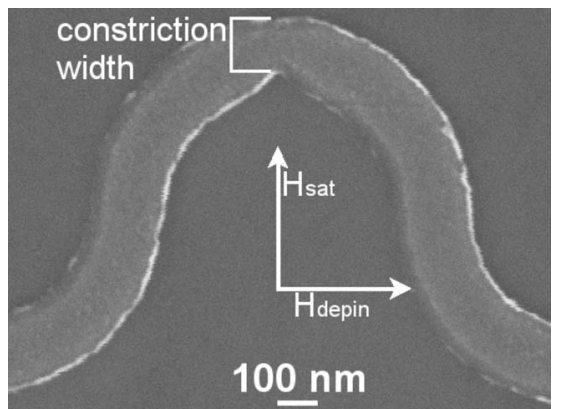

FIG. 1. SEM image of a $200 \mathrm{~nm}$ wide wavy line structure with a small notch (140 $\mathrm{nm}$ constriction). The direction of the saturation field, used to position the domain wall at the notch and the direction of the depinning field, used to move the wall away from the notch, are indicated.

would be hard to lift off. For MR measurements, $200 \mathrm{~nm}$ wide permalloy ring structures with similar constrictions and nonmagnetic contacts have been fabricated. ${ }^{11}$ Standard fourprobe ac lock-in MR measurements were carried out at $4 \mathrm{~K}$. The presence or absence of a domain wall at a constriction can be sensed due to the anisotropic MR contribution of a domain wall. ${ }^{10,11}$ By applying a field while monitoring the resistance, the domain-wall depinning fields are determined. To image these domain walls inside constrictions down to 35 $\mathrm{nm}$ width, very high spatial-resolution (sub-10 $\mathrm{nm}$ ) imaging is necessary, which is provided by off-axis electron holography. ${ }^{14}$ A high-energy (300 kV) electron wave passing through the sample acquires a phase shift, resulting from the presence of electric and magnetic fields. The phase shift is measured by using an electron biprism so that the transmitted electron wave interferes with a wave that passes only through the SiN membrane. This phase shift is then interpreted to obtain the magnitude and direction of the magnetic induction in the specimen.

In order to probe the pinning strength, we have measured the external magnetic field necessary to depin a domain wall from the constriction as a function of the constriction width. The thickness was chosen to be $34 \mathrm{~nm}$ for comparison with earlier experiments. ${ }^{10,11,15}$ The ring structure is first saturated with an in-plane field along the notch position in order to position the domain wall at the constriction and the field is then set to zero (see Fig. 1). Then, a perpendicular in-plane field is applied, and the field necessary to depin the wall is determined (black squares in Fig. 2). The depinning field of the narrowest constrictions ( $335 \mathrm{Oe})$ is about six times the

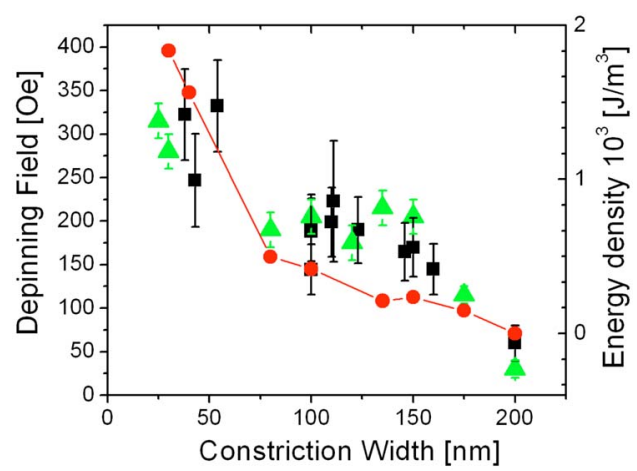

FIG. 2. (Color online) Experimental depinning field (left ordinate) vs constriction width in $200 \mathrm{~nm}$ wide permalloy ring structures with triangular constrictions and a film thickness of $34 \mathrm{~nm}$ (black squares); numerical simulations (green up triangles). The calculated energy density differences show the same trend (red disks, right ordinate).

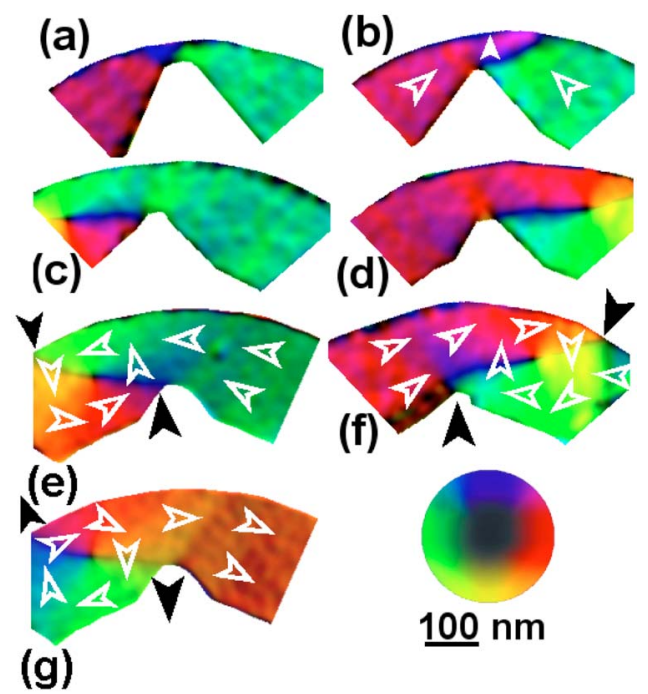

FIG. 3. (Color online) High spatial-resolution electron holography images of sections of $27 \mathrm{~nm}$ thick, $200 \mathrm{~nm}$ wavy line structures with different constriction widths: (a) $35 \mathrm{~nm}$, (b) $60 \mathrm{~nm}$, (c) $100 \mathrm{~nm}$, (d) $110 \mathrm{~nm}$, (e) 140 $\mathrm{nm}$, (f) $160 \mathrm{~nm}$, and (g) $140 \mathrm{~nm}$. The walls in constrictions (a) and (b) are transverse walls, (c) and (e) exhibit vortex head-to-head walls to the left and (d) and (f) vortex head-to-head walls to the right of the constriction; (e) and (g) show the same constriction with (e) a head-to-head and (g) a tail-to-tail vortex wall. The magnetization directions are indicated in (b), (e), (f), and (g) by white arrows. Areas where stray field occurs are denoted with black arrows in (e), (f) and (g). The color code indicating the magnetization direction is also shown.

field needed to move a wall in a ring without a constriction (60 Oe), where the depinning field is governed by material imperfections and the edge roughness. Therefore, the geometrically induced pinning is by far stronger than the pinning due to natural imperfections, which means that the pinning strength can be tailored over a wide range of values by adjusting the notch geometry. The maximum depinning field is much lower than the field needed to nucleate a domain wall $(\approx 600 \mathrm{Oe}),{ }^{15}$ so that we indeed probe the depinning of a domain wall and not the annihilation by a new wall, nucleated at a different position in the ring.

In general, the depinning field increases with decreasing constriction width, indicating that walls in narrower constrictions are more strongly pinned. Qualitatively, this behavior is expected, since the energy of the domain wall scales with the size, so that smaller domain walls in narrower constrictions are energetically more favorable and thus more strongly pinned. However, this simple picture fails to explain why transverse walls are attracted into constrictions, ${ }^{10}$ while vortex walls are repelled at the precise location of the constriction, but pinned close by with a definite sense of rotation (clockwise or counterclockwise depending on which side of the constriction the wall is located). ${ }^{11}$ We now employ direct imaging of domain walls to reveal the details of the interaction of domain walls with constrictions. In Fig. 3, we present high spatial-resolution magnetic induction maps of domain walls at various constrictions, which were prepared by saturating the sample with a field along the notch position and then relaxing the field to zero. For narrow constrictions [Figs. 3(a) and 3(b)] transverse walls can be discerned inside the constrictions, while for larger constrictions [Figs. $3(\mathrm{c})-3(\mathrm{~g})]$ vortex walls are located at positions adjacent to the constrictions (on the left- or right-hand side depending on slight geometrical asymmetries in the notch with respect to the applied field direction). We can now directly correlate the 
sense of rotation with the position with respect to the notch, which is not possible using MR-measurements. We see that the head-to-head walls to the left of the constriction have counterclockwise sense of rotation [Figs. 3(c) and 3(e)], while the walls to the right of the notch have a clockwise sense of rotation [Figs. 3(d) and 3(f)]. When the field is reversed to create tail-to-tail walls, the vortex wall sense of rotation is reversed. This can be seen by comparing the vortex walls to the left of the notch in Figs. 3(e) and 3(g), which show the same notch after applying opposite fields. This is a universal behavior, which we observed for all vortex walls at constrictions for all film thicknesses. To understand this behaviour, we have to take into account the fact that a vortex wall has two areas where a stray field occurs. In Fig. 3, the positions at which the stray field enters [Figs. 3(e) and 3(f)] or leaves [Fig. $3(\mathrm{~g})]$ the vortex wall are marked by two black arrows. In order to minimize the stray field associated with the vortex wall, the lower of the two stray field areas is always located inside the notch, which determines the sense of rotation of the vortex wall. As a result, the wall is actually pinned in the location directly adjacent to the notch (even though it is repelled from the constriction). This behavior explains the fact that in Fig. 2, a significant depinning field is also necessary to displace the vortex walls at wide constrictions. ${ }^{16}$

Having established the details of the domain-wall behavior at constrictions, we now turn to the physics behind the strength of the domain-wall pinning. To fully understand our experimental depinning field results, we have carried out micromagnetic simulations, using the OOMMF package. ${ }^{17}$ The intrinsic parameters used are: $M_{S}=800 \times 10^{3} \mathrm{~A} / \mathrm{m}, A=13$ $\times 10^{-12} \mathrm{~J} / \mathrm{m}$, and a cell size of $5 \mathrm{~nm}$. Since the domain-wall types obtained from OOMMF simulations $(0 \mathrm{~K})$ do not always correspond to the experimentally observed wall type, ${ }^{18}$ we have inserted the type of domain-wall determined experimentally at this constriction (as described in Ref. 11) into the simulation. ${ }^{19}$ To obtain the theoretical depinning fields, we have simulated the experiment by relaxing the experimentally observed domain-wall type in the constriction at the notch position and then applying a perpendicular field until the wall depins. The simulated depinning fields (triangles in Fig. 2) reproduce the experiment (black squares in Fig. 2) very well. They are highest for the transverse walls inside the constriction (20-40 nm constriction width) and are lower and vary less for vortex walls adjacent to constrictions with different sizes (80-175 nm constriction width) due to the different pinning mechanisms of the two different wall spin structures. The nonzero depinning field without a notch (200 $\mathrm{nm}$ width) is due to the edge roughness induced by the cubic cell discretization.

In order to understand the pinning further, we now discuss the energetics that govern the domain-wall pinning. To depin a wall, the depth of the energy well has to be overcome by the external field and as a simple model the depth is given by the energy difference of a wall inside the constriction and when it is moved outside, which we determine from the energy densities of the different walls at different positions, calculated using the OOMMF package as shown in Fig. 2. ${ }^{10}$ We see that the energy density differences exhibit a very similar dependence on the constriction width as the depinning fields. This observation suggests that the experimentally obtained depinning fields are a measure of the energy barrier height, because as a first approximation, the extra Zeeman energy for the domain wall due to the perpendicularly applied depinning field is proportional to the applied field strength. Since we can now obtain the depth of the potential well (strength of the pinning) as well as its width (as described in Ref. 10), our measurements open the way to artificially engineer the potential landscape, as required for any application based on domain walls.

In conclusion, we have determined the spin structure of geometrically confined head-to-head domain walls with sub-10 nm spatial resolution. We observe a direct correlation of the spin structure with the constriction width and the wall position with respect to the constriction. Using MR measurements, we have systematically determined the domain-wall depinning fields in constrictions down to $35 \mathrm{~nm}$ width, with transverse walls exhibiting significantly higher depinning fields than vortex walls due to different pinning mechanisms. Correlation with the measured depinning fields allows us to infer that the depinning field can be used as an experimental measure of the depinning energy barrier height.

The authors acknowledge support by the Deutsche Forschungsgemeinschaft (SFB 513), the CMI Magnetoelectronic Devices project, the Landesstiftung BadenWürttemberg, the "Deutscher Akademischer Austauschdienst" (DAAD) and M. Bolte for help with micromagnetic simulations. One of the authors (D. B.) acknowledges support by the European Union (Interreg III A program) and another author (R. E. D.-B.) acknowledges support by the Royal Society.

${ }^{1}$ D. A. Allwood, G. Xiong, M. D. Cooke, C. C. Faulkner, D. Atkinson, N. Vernier, and R. P. Cowburn, Science 296, 5575 (2002).

${ }^{2}$ A. Yamaguchi, T. Ono, S. Nasu, K. Miyake, K. Mibu, and T. Shinjo, Phys. Rev. Lett. 92, 077205 (2004).

${ }^{3}$ M. Kläui, C. A. F. Vaz, J. A. C. Bland, W. Wernsdorfer, G. Faini, E. Cambril, L. J. Heyderman, F. Nolting, and U. Rüdiger, Phys. Rev. Lett. 94, 106601 (2005).

${ }^{4}$ L. Lopez-Diaz, M. Kläui, J. Rothman, and J. A. C. Bland, Physica B 306, 211 (2001).

${ }^{5}$ J. Grollier, D. Lacour, V. Cros, H. Hamzic, A. Vaures, A. Fert, D. Adam, and G. Faini, J. Appl. Phys. 92, 4825 (2002).

${ }^{6}$ M. Kläui, C. A. F. Vaz, A. Lapicki, T. Suzuki, Z. Cui, and J. A. C. Bland, Microelectron. Eng. 73, 785 (2004).

${ }^{7}$ J. Grollier, P. Boulenc, V. Cros, A. Hamzić, A. Vaurès, and A. Fert, Appl. Phys. Lett. 83, 509 (2003).

${ }^{8}$ D. A. Allwood, G. Xiong, and R. P. Cowburn, Appl. Phys. Lett. 85, 2848 (2004).

${ }^{9}$ A. Himeno, T. Okuno, S. Kasai, T. Ono, S. Nasu, K. Mibu, and T. Shinjo, J. Appl. Phys. 97, 66101 (2005).

${ }^{10}$ M. Kläui, C. A. F. Vaz, J. Rothman, J. A. C. Bland, W. Wernsdorfer, G. Faini, and E. Cambril, Phys. Rev. Lett. 90, 097202 (2003).

${ }^{11}$ M. Kläui, C. A. F. Vaz, W. Wernsdorfer, E. Bauer, S. Cherifi, S. Heun, A. Locatelli, G. Faini, E. Cambril, and L. J. Heyderman, and J. A. C. Bland, Physica B 343, 343 (2004).

${ }^{12}$ A. J. Zambano and W. P. Pratt Jr., Appl. Phys. Lett. 85, 1562 (2004).

${ }^{13}$ L. J. Heyderman, M. Kläui, R. E. Schäublin, U. Rüdiger, C. A. F. Vaz, J. A. C. Bland, and C. David, J. Magn. Magn. Mater. 290, 86 (2005).

${ }^{14}$ R. E. Dunin-Borkowski, M. R. McCartney, B. Kardynal, S. S. P. Parkin, M. R. Scheinfein, and D. J. Smith, J. Microsc. 200, 187 (2000).

${ }^{15}$ M. Kläui, C. A. F. Vaz, J. A. C. Bland, E. H. C. P. Sinnecker, A. P. Guimaraes, W. Wernsdorfer, G. Faini, E. Cambril, L. J. Heyderman, and C. David, Appl. Phys. Lett. 84, 951 (2004).

${ }^{16}$ In the MR experiment, the vortex walls were deliberately positioned on one side of the constriction, so that the walls were pulled away from the notch, not through the constriction.

${ }^{17}$ The free oOMmF package is available at http://math.nist.gov/oommf.

${ }^{18}$ M. Kläui, C. A. F. Vaz, J. A. C. Bland, L. J. Heydermand, F. Nolting, A. Pavlovska, E. Bauer, S. H. S. Cherifi, and A. Locatelli, Appl. Phys. Lett. 85, 5637 (2004).

${ }^{19}$ M. Laufenberg, M. Kläui, and U. Rüdiger (unpublished). 